\title{
Dual-Energy CT for Pulmonary Embolism: Current and Evolving Clinical Applications
}

\author{
Yoo Jin Hong ${ }^{1}$, Jina Shim ${ }^{1}$, Sang Min Lee ${ }^{2}$, Dong Jin Im $^{1}$, Jin Hur ${ }^{1}$ \\ ${ }^{1}$ Department of Radiology and Research Institute of Radiological Science, Severance Hospital, Yonsei University College of Medicine, Seoul, Korea; \\ ${ }^{2}$ Department of Radiology and Research Institute of Radiology, Asan Medical Center, University of Ulsan College of Medicine, Seoul, Korea
}

Pulmonary embolism (PE) is a potentially fatal disease if the diagnosis or treatment is delayed. Currently, multidetector computed tomography (MDCT) is considered the standard imaging method for diagnosing PE. Dual-energy CT (DECT) has the advantages of MDCT and can provide functional information for patients with PE. The aim of this review is to present the potential clinical applications of DECT in PE, focusing on the diagnosis and risk stratification of PE.

Keywords: Dual-energy computed tomography; Pulmonary embolism; Iodine; Pulmonary artery sarcoma; Chronic

thromboembolic pulmonary hypertension

\section{INTRODUCTION}

Pulmonary embolism (PE) is a potentially fatal disease with varying clinical presentations. It is the third most common cause of death in the United States. It can have a fatal prognosis if the diagnosis or treatment is delayed. Accordingly, prompt diagnosis, in turn allowing for prompt treatment, can dramatically reduce the mortality rate [1-5].

Currently, multidetector computed tomography (MDCT) is considered the standard imaging modality for diagnosing $\mathrm{PE}$ [6]. Technical advancements in CT in the past decade have allowed physicians to obtain images of higher quality, with exposure to lower levels of radiation, which demonstrate small subsegmental pulmonary artery (PA) embolisms $[7,8]$. CT has high diagnostic accuracy in detecting PE [912]. It is the most clinically important imaging modality for evaluating patients with PE. In addition, it can provide

Received: December 30, 2020 Revised: February 22, 2021 Accepted: March 19, 2021

Corresponding author: Jin Hur, MD, PhD, Department of Radiology and Research Institute of Radiological Science, Severance Hospital, Yonsei University College of Medicine, 50 Yonsei-ro, Seodaemun-gu, Seoul 03722, Korea.

- E-mail: khuhz@yuhs.ac

This is an 0pen Access article distributed under the terms of the Creative Commons Attribution Non-Commercial License (https://creativecommons.org/licenses/by-nc/4.0) which permits unrestricted non-commercial use, distribution, and reproduction in any medium, provided the original work is properly cited. crucial information on the hemodynamic stability and prognosis of patients with acute PE [13-15].

Dual-energy computed tomography (DECT) can provide both morphological and functional pulmonary information of the lung in a single contrast-enhanced examination. It has the advantages of MDCT and uses $\mathrm{X}$-rays with two different energy spectra to detect specific substances according to the material decomposition theory $[16,17]$. This enables selective visualization of the iodine components in tissues. It has recently been actively applied in patients with PE owing to the simultaneous demonstration of pulmonary emboli occluding the PA and resultant perfusion defects in the lung parenchyma [18-20]. This may yield more information about the burden of PE because both perfusion and angiographic images are obtained without increasing the radiation dose. Thus, DECT is a feasible option for the evaluation of PE [21,22].

The aim of this review is to discuss the potential clinical applications of DECT in PE, focusing on the diagnosis and risk stratification of $\mathrm{PE}$.

\section{Diagnosis of PE Based on DECT: Can DECT Increase the Diagnostic Accuracy of PE?}

DECT is useful for detecting acute PE [20,22-25]. The main strength of DECT is that it can show perfusion defects in the lung parenchyma caused by PA occlusion and enable 
direct visualization of filling defects in the PAs $[26,27]$. The iodine map provides a quick approach to perfusion defects and enables quantitative analysis of the volume of perfusion defects. The quantitative assessment of pulmonary perfusion strongly correlates with pulmonary perfusion scintigraphy and single-photon emission CT (SPECT) images $[28,29]$.

Many small-scale studies have reported the diagnostic value of DECT for the detection of acute PE. The reported sensitivity and specificity range from $60 \%$ to $90 \%$ and $88 \%$ to $99 \%$, respectively $[24,25,27,29-32]$. In chronic PE, DECT has $96-100 \%$ sensitivity and $76-96 \%$ specificity using SPECT or scintigraphy as a reference $[33,34]$.

Recent studies have focused on the added values of DECT over CT pulmonary angiography (CTPA) $[26,35]$. In their study, the diagnostic accuracy of CTPA (85.5-90.4\%) improved for both readers with a combination of colorcoded lung-perfused blood volume images (95.6-97.6\%) with high interobserver and intraobserver agreements (Table 1) [35]. Moreover, the addition of DECT to CTPA improved the detection of peripheral intrapulmonary clots [35]. Small peripheral clots are occasionally difficult to distinguish from the surrounding segmental or subsegmental pulmonary arteries on MDCT. However, DECT is useful for identifying regional iodine perfusion defects due to small clots (Fig. 1). In a recent large-scale retrospective study involving 1035 consecutive patients, a DECT iodine map helped to detect small (segmental or subsegmental) pulmonary emboli in $1 \%$ of additional patients [26]. In addition, the DECT technique can improve the image quality even with a small amount of contrast agent (Fig. 2). Using monoenergetic image reconstruction, the vessel attenuation and contrast-to-noise ratio can be improved [36]. This means that when using DECT in PE imaging, the amount of iodine contrast can be reduced, while still maintaining adequate image quality with a high diagnostic confidence.

A recent study reported that deep learning-based automatic lung lobe segmentation and quantitative lobar or zonal perfusion analyses are useful in PE detection, although the study had a small sample size of 88 patients [37]. Further large-scale studies are needed to assess whether deep learning-based quantitative lobar perfusion parameters can provide complementary diagnostic or prognostic information in patients with PE undergoing DECT.

\section{Ventilation CT Using DECT in PE}

Many studies have reported the usefulness of xenon- enhanced ventilation $\mathrm{CT}$ in patients with pulmonary disease [38-40]. Ventilation/perfusion imaging using DECT can visualize the ventilation/perfusion match or mismatch in patients with suspected PE. Xenon is a radiodense gas with a high atomic number of 54, similar to iodine (atomic number: 53). It exhibits photoelectric absorption properties similar to iodine and can therefore be isolated from lung tissues using the material decomposition method.

A previous study performed DECT ventilation/perfusion imaging in 32 patients with suspected PE. Of the 32 patients, six reported mild adverse events (i.e., shortness of breath, mild dizziness, and limb numbness). DECT lung ventilation/perfusion imaging was successfully conducted in 10 patients with $\mathrm{PE}$, and a PE-related ventilation/ perfusion mismatch was found in eight patients. The authors concluded that DECT lung ventilation/perfusion imaging could provide high-spatial-resolution information on morphological and functional ventilation/perfusion of the lungs in patients with suspected PE [41].

However, because the atomic number of xenon is close to that of iodine, xenon cannot be distinguished from iodine. Therefore, perfusion and ventilation imaging using iodine and xenon cannot be performed using a single imaging procedure. In their study, 5 minutes after the xenonenhanced DECT imaging examination, dual-energy CTPA was performed [41]. Therefore, in another study, alternative inhaled contrast agents were suggested for simultaneous ventilation-perfusion imaging for PA-occlusive diseases. In a preclinical study, a ventilation-perfusion evaluation method using krypton (atomic number: 34 ) and iodine was suggested [42]. Although ventilation scans obtained with xenon or krypton are limited to use in research, they have the potential to become a non-invasive tool for examining the anatomical structures of the lungs and lung ventilation.

\section{DECT for Differentiation of PE and PA Sarcoma}

PA sarcoma is a rare type of malignancy that arises from the intima or media of the PA and is frequently misdiagnosed as a PE because clinical symptoms and radiological findings are often similar. Early diagnosis and radical resection of PA sarcomas are crucial for survival. Therefore, it is important to clinically differentiate PA sarcomas from PE. CT is an imaging modality that allows for the differential diagnosis of PA sarcoma and PE. It can help differentiate PA sarcoma from PE by visualizing extraluminal tumor extension, expansion of the involved 


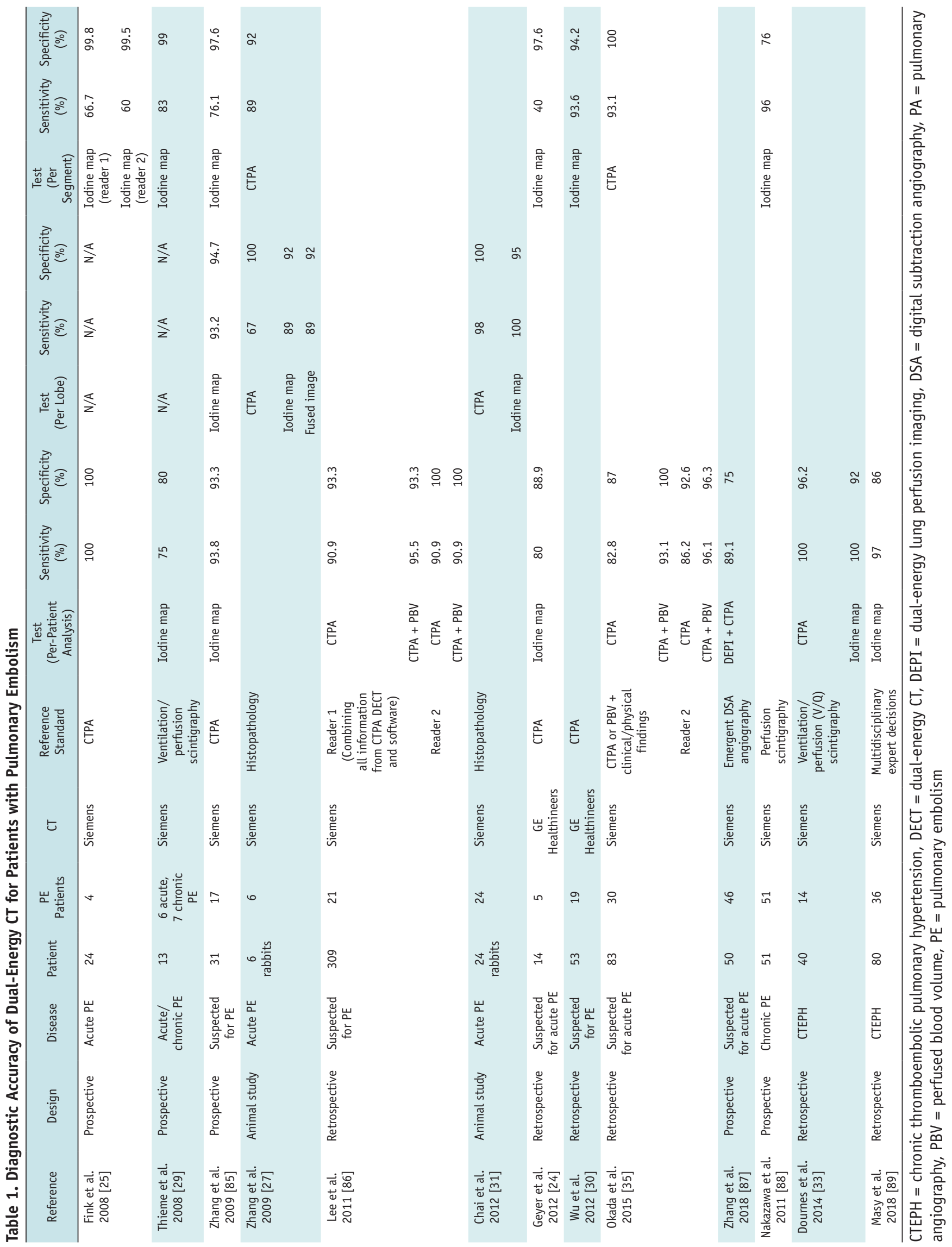



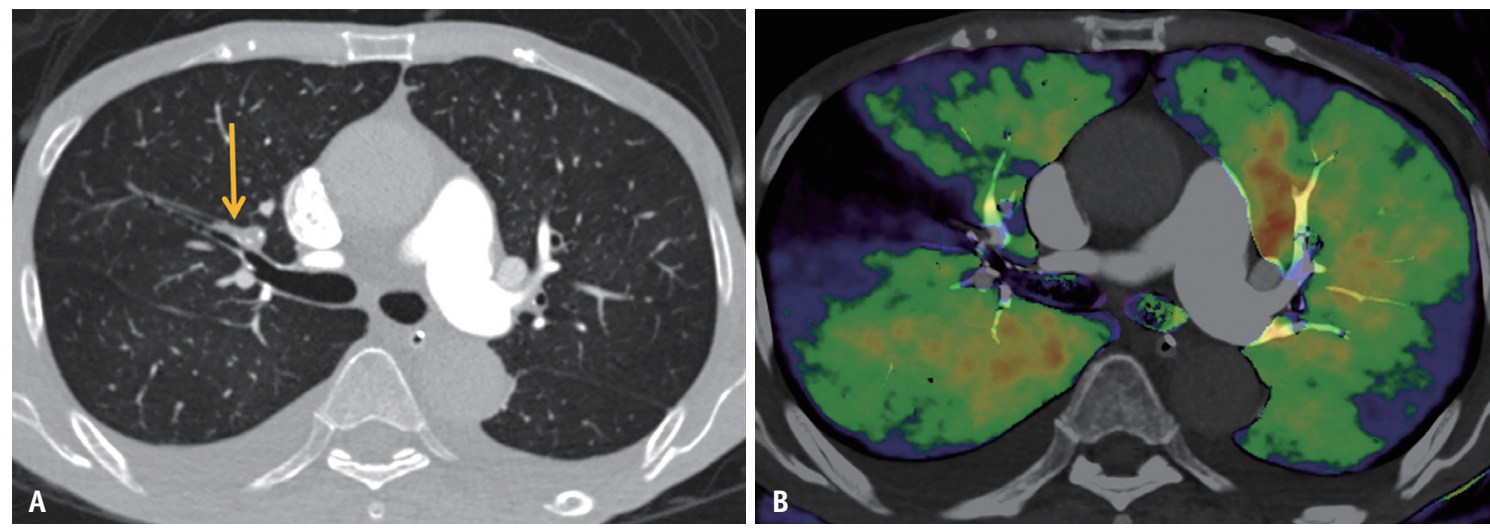

Fig. 1. A 76-year-old female diagnosed with acute pulmonary thromboembolism.

A. CT angiography showing a focal filling defect in the right upper lobar pulmonary artery (arrow) due to acute pulmonary thromboembolism and bilateral pleural effusion. B. The fusion image of CT angiography and a color-coded iodine map showing a thrombus in the right upper pulmonary artery as well as a corresponding wedge-shaped perfusion defect in the right upper lobe.
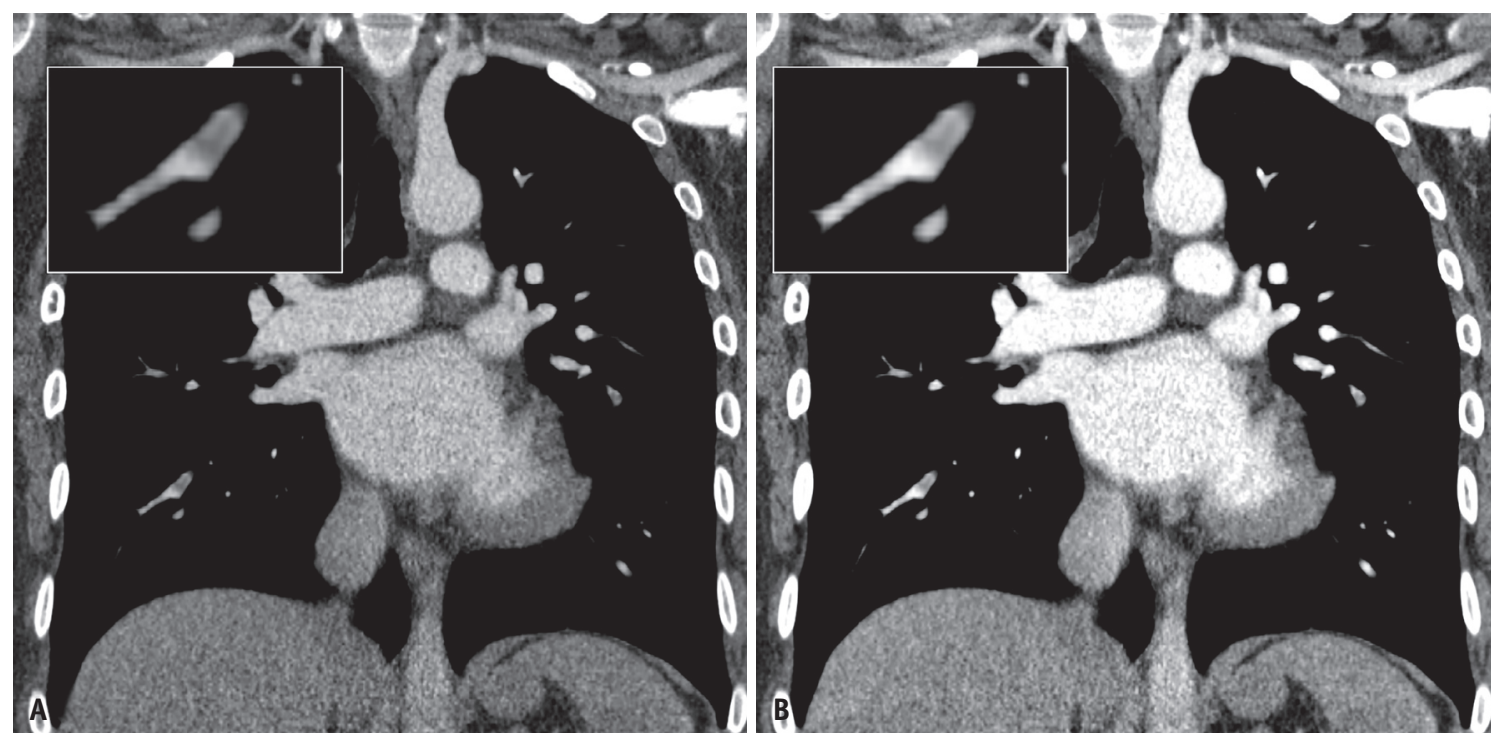

Fig. 2. A 30-year-old female diagnosed with acute pulmonary thromboembolism.

A. The conventional image with $100 \mathrm{kVp}$ showing a focal filling defect in the right lower subsegmental artery. B. A monoenergetic image with 60 keV better depicting a subsegmental thrombus in the right lower lobe.

arteries, or a low-attenuation filling defect of the proximal or main PA. However, these findings might not appear in early-stage PA sarcoma, and in cases of extensive PE, the imaging findings can be similar. Because a dual-energy technique allows iodine to be differentiated from other materials, DECT can help differentiate PE and PA sarcoma by detecting subtle lesion enhancements (Fig. 3). A previous study compared quantitative parameters of DECT between 19 PE and six PA sarcoma cases and found no difference in CT Hounsfield units (HU) (PE vs. PA sarcoma: $45.5 \pm$ 15.9 HU vs. $47.1 \pm 9.2 \mathrm{HU} ; p=0.776$ ), but a significant difference in the iodine concentration measured on an iodine map (PE vs. PA sarcoma: $0.6 \pm 0.4 \mathrm{mg} / \mathrm{mL}$ vs. $1.5 \pm$
$0.6 \mathrm{mg} / \mathrm{mL} ; p=0.001)$ [43]. These findings showed the possibility that quantification of iodine values using DECT could help differentiate PE from PA sarcoma. Generally, fluorodeoxyglucose (FDG) PET-CT is a useful method to distinguish PA sarcoma from PE based on FDG uptake with a difference in the maximum standard uptake value [4447]. However, PE can have varying uptake, which results in difficulties in differentiating it from PA sarcoma $[48,49]$. DECT has the added roles of specific tissue characterization, clear anatomical delineation, and simultaneous evaluation of lung perfusion [43]. 

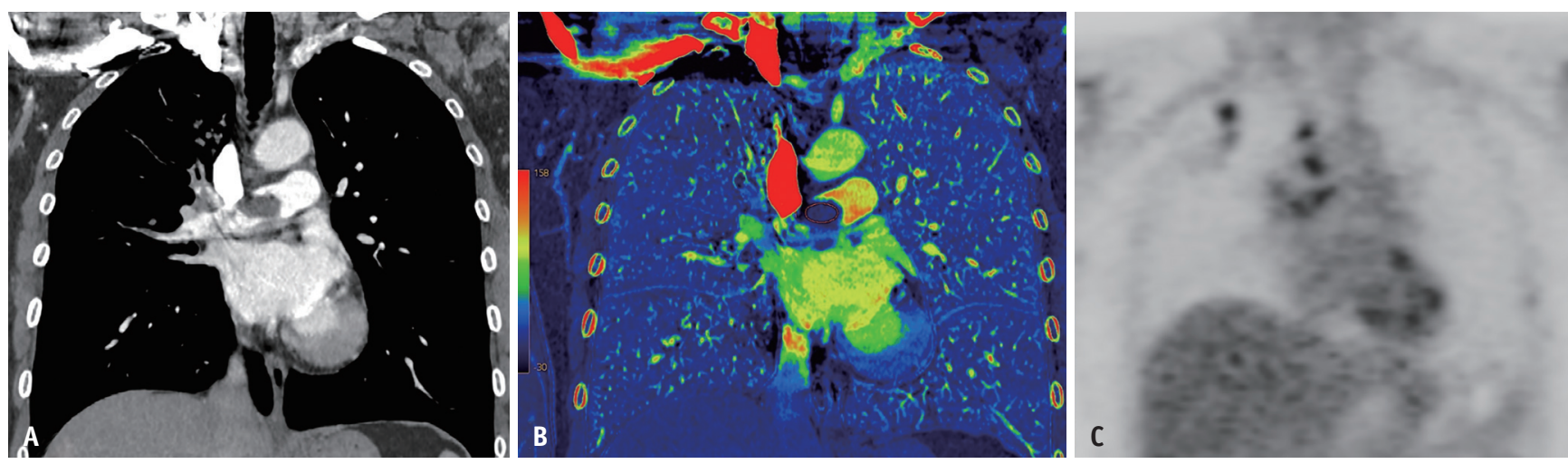

Fig. 3. A 58-year-old female with pulmonary artery sarcoma.

A. A coronal contrast enhanced CT image showing an intraluminal mass with an eccentric filling defect in the right main pulmonary trunk. B. 0n a coronal color-coded iodine (water) image from dual-energy CT, the mean iodine concentration within the region of interest is $2.6 \mathrm{mg} / \mathrm{mL}$.

C. A coronal PET image showing focal fluorodeoxyglucose uptake in the intraluminal mass and right upper paratracheal lymph nodes.

\section{DECT in Assessing Severity and Predicting the Prognosis of Patients with PE}

Risk stratification is important in patients with PE because optimal management, monitoring, and therapeutic strategies depend on prognosis. Many CT parameters have been proposed as potential predictors of PE severity and clinical outcomes $[14,15,32,50,51]$. Among them, the quantitative CT parameter of right ventricular (RV) dysfunction, or an abnormally increased ratio between the RV and left ventricular (LV) diameter in transverse CT sections, is proposed as a strong predictor of adverse clinical outcomes in patients with acute PE [15].

Recently, DECT has been performed for severity assessment and prediction of clinical outcomes in patients with PE [52,53]. Several studies have shown the feasibility of DECT to assess perfusion defects in pulmonary blood volume (PBV) caused by PE [35,37]. Although data are limited regarding the functional relevance of perfusion changes detected with DECT, a few studies have shown a higher rate of right-heart strain among patients with acute PE presenting with a greater extent of perfusion defects on DECT. Previous studies have also shown that the extent of perfusion defects on DECT correlates with adverse clinical outcomes among patients with PE $[52,53]$.

The perfusion defect score calculated using DECT has been suggested as a new imaging biomarker for severity assessment in patients with PE. In a previous study, perfusion defect scores based on DECT scans were used to assess the severity of PE in 30 patients, and the scores were found to correlate well with the RV/LV diameter ratio $(r=0.69, p<0.001)$ and PA obstruction score $(r=0.87$, $p<0.001)$ [54]. In another study involving 55 patients with PE [55], perfusion defect scores calculated using DECT significantly correlated with the CTPA obstruction score and RV/LV ratio $(r=0.62$ and 0.60 , respectively, $p<0.001)$ and effectively differentiated between the low- and intermediate-risk groups $(p=0.011)$ [55]. Recently developed software provides a quick automated quantification of pulmonary perfused blood volume, which correlates well with the severity of PE [56].

A pilot study suggested that the volume of the lung with perfusion defects measured using DECT may be used not only as a surrogate marker, but also as a prognosticator of right heart strain in patients with acute PE $[52,53]$. Another study involving 60 patients with PE reported that the extent of perfusion defects measured on a DECT iodine map is a predictor of adverse clinical outcomes (death or intensive care treatment within 60 days). A multicenter retrospective study involving 115 patients with suspected PE without a detectable thromboembolic clot found that the volume of the lung with perfusion defects is a predictor of patient prognosis [57].

Based on previous studies, quantitative DECT parameters have the potential to be used as prognostic markers in acute PE (Fig. 4) [52,53]. However, existing studies were performed with small groups of participants and lacked the additional effectiveness of perfusion defect quantification with DECT compared to the CT ventricular diameter ratio, which is a well-established and widely used prognostic indicator. In addition, the value of quantitative DECT parameters for prognosis and risk stratification in acute PE is controversial. A recent study involving 172 patients with acute PE demonstrated that while the RV/LV ratio predicted 

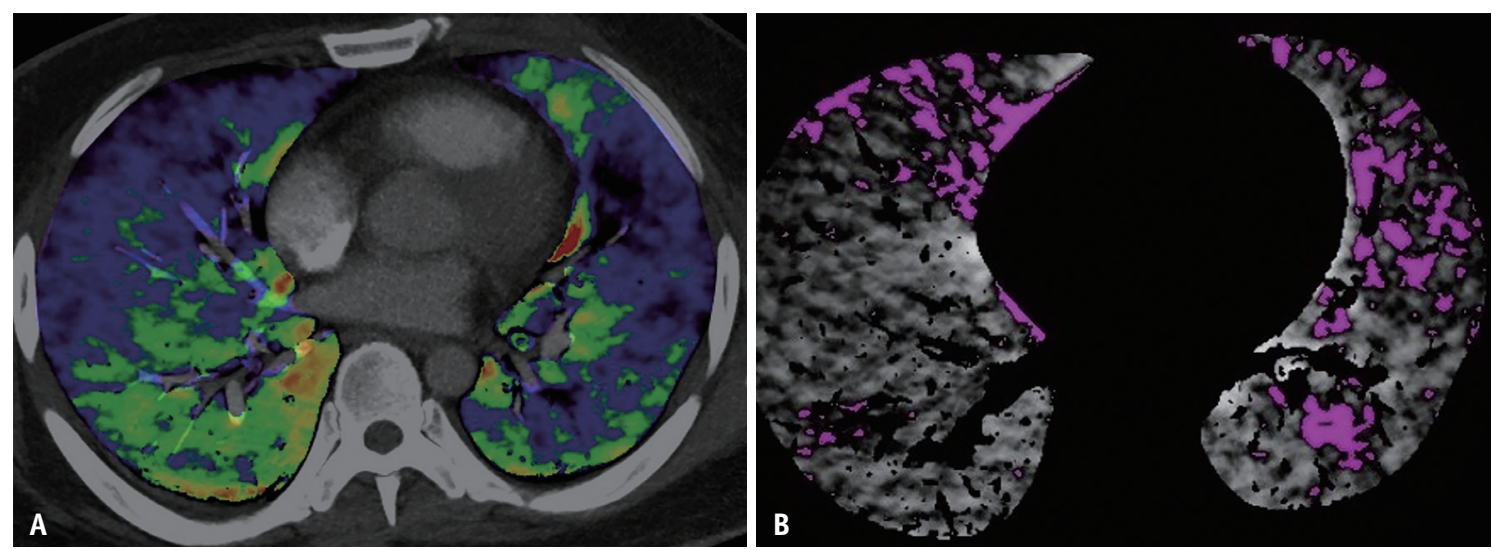

Fig. 4. A 34-year-old male with acute pulmonary embolism.

A. A color-coded iodine map showing multifocal perfusion defects in both upper lobes due to pulmonary thromboembolisms. B. A map obtained with a volume analysis software showing a perfusion defect volume, measured from -1024 to -1 Hounsfield units, of $324.15 \mathrm{~cm}^{3}$ and a relative perfusion defect volume of $10.24 \%$.

30-day mortality (hazard ratio, 3.8; $p=0.002$ ) and PErelated death (hazard ratio, $18.1 ; p<0.001$ ), perfusion defects detected using DECT had no added benefit for predicting 30-day mortality over the RV/LV ratio [58]. We conducted a propensity score-matched study to compare the predictive value of quantitative DECT parameters and CT ventricular diameter ratio in patients with acute PE. The propensity-matched study population included 240 patients with acute PE in the CTPA group and 240 patients with acute $\mathrm{PE}$ in the DECT group. According to the results, lung perfusion defects measured with DECT had no added benefit over the CT RV/LV ratio alone for prediction of death within 30 days ( $C$-statistics: $0.80,0.83, p=0.097$ ) [59]. Currently, the data do not prove any additional benefit of functional lung assessment with DECT to predict death from PE [59]. Therefore, larger trials with longer follow-up periods should be performed to estimate the potential influence of DECT findings on treatment strategies to optimize the treatment and outcome of patients with acute PE.

\section{DECT and Chronic PE and Chronic Thromboembolic Pulmonary Hypertension (CTEPH)}

If acute PE does not dissolve over time, it can result in chronic PE. Once emboli attain a chronic organized status, secondary hemodynamic changes develop, eventually causing chronic pulmonary hypertension, followed by right heart failure. This progressive course can be corrected using surgical pulmonary thromboendarterectomy. Therefore, differentiating between acute and chronic emboli is important when pulmonary emboli are detected on $\mathrm{CT}$ images.

Chronic PE has a different perfusion pattern from acute PE because systemic collateral circulation increases to sustain lung tissues distal to the occluded pulmonary arteries (Fig. 5). DECT is useful for demonstrating different perfusion patterns between acute and chronic $P E$. In a previous study, two-phase DECT was performed in 42 patients with $\mathrm{PE}$, and iodine-related attenuation (IRA) change ratios were calculated using the formula $100 \% \times$ ([IRA of delayed phase] - [IRA of PA phase])/(IRA of PA phase). IRA change ratios for patients with acute $\mathrm{PE}$ and those with chronic PE were $-3.14 \%$ and $191.9 \%(p<0.001)$, respectively, indicating that delayed enhanced patterns were observed in chronic PE segments. This may be because extensive systemic collateral formation occurs in chronic PE via the bronchial arteries [60]. These results demonstrated that DECT can display iodine distribution to reflect perfusion patterns [61].

In addition to evaluating parenchymal perfusion defects, DECT data are useful for the detection and tissue characterization of intravascular thrombotic material using the material decomposition method, thus differentiating between acute and chronic PE. Kim et al. [62] compared $\mathrm{HU}$, iodine-related $\mathrm{HU}$ (HU from monochromatic $70 \mathrm{keV}$ images, HU from mass spectroscopy imaging), and iodine concentration of embolism between 15 patients with acute $\mathrm{PE}$ and 11 patients with chronic PE. They found that chronic PE had a significantly higher mean $\mathrm{HU}$, iodine-related $\mathrm{HU}$, and IC compared to acute PE. This may be because the fibrotic components of a chronic thrombus have a greater vascular supply compared to acute thrombi.

Chronic thromboembolic pulmonary hypertension 

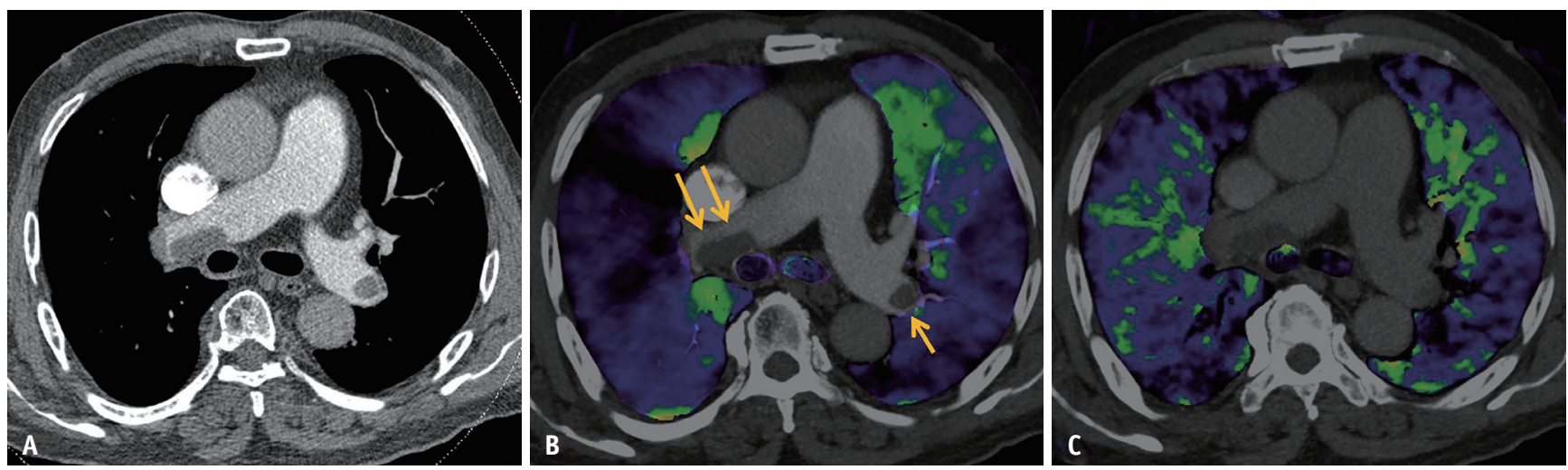

Fig. 5. A 60-year-old male diagnosed with chronic pulmonary thromboembolism.

A. CT angiography image showing organized thrombi in both the main pulmonary arteries. B. In the pulmonary artery phase, a fusion image of CT angiography and color-coded iodine map showing organized thrombi in both main pulmonary arteries (arrows) with a wedge-shaped perfusion defect in the right upper lobe. C. On the delayed-phase image, the area with a previous perfusion defect in the right upper lobe is enhanced due to the systemic collateral supply in chronic pulmonary thromboembolism.

(CTEPH) develops in $2-4 \%$ of patients with acute PE [6368]. Thromboembolic materials in the pulmonary vascular bed trigger vasoconstriction and remodeling $[63,69]$, and progressive pulmonary hypertension and right heart failure can occur [69]. The prognosis of CTEPH is poor. Therefore, accurate diagnosis and prompt treatment are important for a better prognosis $[63,68]$. DECT can play a potential role in the diagnosis and treatment of CTEPH. It is useful for evaluating perfusion patterns and differentiating between CTEPH and PA hypertension. The authors found that $96.6 \%$ of segments from 19 patients with PA hypertension showed patchy perfusion defects (heterogeneous perfusion defects with intervening areas of preserved perfusion), while 12 patients with CTEPH usually showed patchy $(58.5 \%$ of segments) or embolic-type (37.5\% of segments) perfusion defects (wedge-shaped, pleural perfusion defects in the affected segment) on the iodine maps of DECT [70]. DECT is also useful for the severity assessment of CTEPH and evaluation of the treatment response in CTEPH (Fig. 6). Severity assessment and treatment are crucial for CTEPH because untreated CTEPH leads to a poorer prognosis compared to acute PE. A previous study reported that PBV scores obtained using DECT significantly correlated with PA pressure and pulmonary vascular resistance, and lung PBV scores are useful noninvasive estimators of CTEPH [71]. DECT can be used to measure an increase in lung perfusion following balloon pulmonary angioplasty (BAP) in patients with CTEPH [71]. The authors examined the clinical significance of PBV using DECT in eight patients with CTEPH undergoing BPA. They found increases in lung PBV values in the BPA-treated area and significant positive correlations with improvement in whole-lung PBV. This study suggests that DECT may be a useful noninvasive tool for assessing the treatment outcome of BAP.

\section{Technical Consideration and Radiation Dose in DECT}

In terms of radiation dose, initial studies reported that DECT was associated with higher radiation exposure than single-energy CT [72]. However, many studies have reported that DECT does not require the additional dose required for conventional CT $[16,40,73]$. A previous phantom study reported that the radiation doses of DECT pulmonary angiography were $2.61 \mathrm{mSV}$ and $2.69 \mathrm{mSV}$ for $140 / 80 \mathrm{kVp}$ and Sn 140/100 kVp, respectively, which were similar to the 2.70-mSV dose for the standard 120-kVp protocol chest CT [73]. A two-phase imaging method may increase radiation dose. However, dose-reduction techniques, such as selective photon-shield usage and tube-current modulation, would be useful for minimizing the increase in radiation dose $[61,74]$. With the development of third-generation DECT, new levels of dual-energy voltage combination and thicker thin filters with a more precise spectral separation of $\mathrm{X}$-rays allow lower radiation exposure with preserved image quality for CTPA examination than that with second-generation DECT or single-energy CT [75-77].

As mentioned above, many studies have used the perfusion defect size on DECT as a surrogate marker that represents disease severity or prognosis. However, the size of the perfusion defect varies depending on various factors (e.g., amount, concentration, injection rate of contrast 

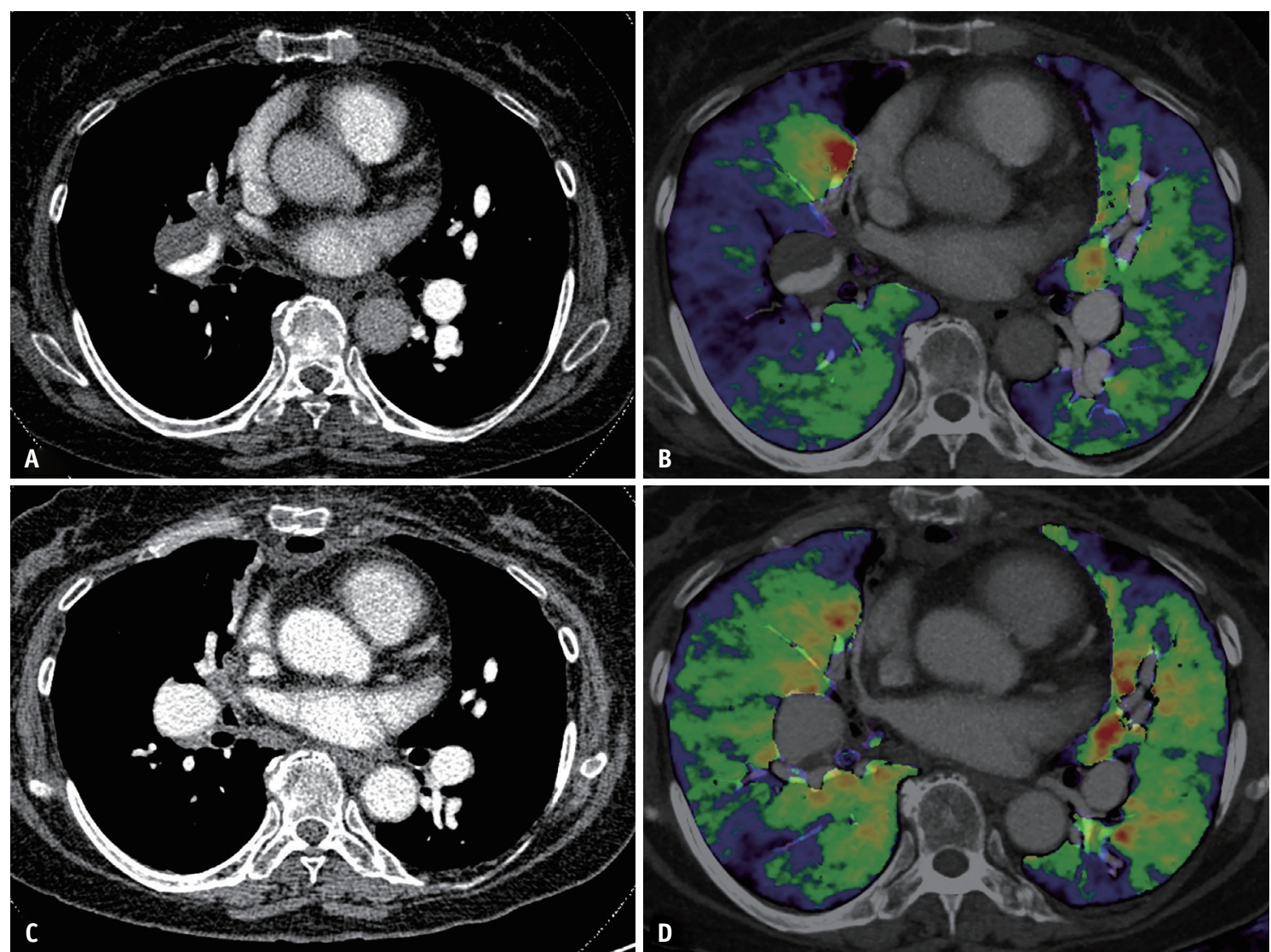

Fig. 6. A 74-year-old female with chronic pulmonary embolism.

A. CT angiography showing an eccentric chronic thrombus in the right lower pulmonary artery. B. The fusion image of CT angiography and colorcoded iodine map showing a large eccentric chronic thrombus in the dilated right lower lobar pulmonary and multifocal perfusion defects in both lungs. C, D. After pulmonary endarterectomy, the organized thrombus was removed. The follow-up CT angiography image showing the contrastenhanced right lower pulmonary artery after removal of the organized thrombus. An iodine map image demonstrates improved pulmonary perfusion on both lungs.

media, tube voltage, scan delay time, and machine). In addition, quantitative measurements are another major issue in DECT imaging because there are no standardized analytical methods or parameters in DECT. Therefore, several values need to be validated for applications, and some values are difficult to analyze.

As such, a scanning protocol should be considered. Currently, there is no standardized scanning protocol for evaluating perfusion defects on DECT. Several studies have proposed an image acquisition and contrast injection protocol to evaluate pulmonary disease using DECT $[21,78]$. Usually, an 80-kVp image is recommended for the lower tube voltage to better demonstrate small endoluminal clots $[21,78]$. To use contrast agents, a high-concentration iodine-based contrast material $(>300 \mathrm{mg} / \mathrm{mL}$ ) is recommended to differentiate iodine from other materials. To inject the contrast media, the region of interest is usually placed in the main PA, and the bolus tracking method is used. To evaluate the PA and lung parenchyma in a single scan, the delayed scan time should be longer (4-7 seconds). The scan direction should be caudocranial to avoid streaky artifacts caused by high-concentration contrast agents in the superior vena cava or upper thoracic veins. The use of saline chasers helps reduce artifacts and improve image quality. If chronic PE is suspected, a delayed-phase scan can be added to evaluate the perfusion pattern, but there is a concern regarding additional radiation exposure.

To achieve optimal parenchymal enhancement, the iodine injection technique, delay time for image acquisition, direction of $\mathrm{CT}$ data acquisition, and reduction of artifacts should be considered. We recommend the use of highconcentration iodine-based contrast agents, prolonged delayed scan time, caudocranial scanning direction, and single-phase scanning. 


\section{Diagnostic Pitfalls of DECT}

Familiarizing clinicians with diagnostic pitfalls of DECT perfusion imaging is important to avoid misdiagnosis. An iodine map of the lung parenchyma allows for the fast detection of perfusion defects and has high inter-reader agreement (Fig. 7). However, an iodine map must be interpreted with caution because iodine maps selectively demonstrate iodinated contrast material within the lung parenchyma, not true perfusion [32]. In addition, PE does not always cause perfusion defects in an iodine map $[20,25,34,79]$. Although the rate of perfusion defects on PBV for occlusive PE is relatively high (82-95\%), the rate of perfusion of non-occlusive PE defects may be as low as $6-9 \%[20,25,34]$, and other lung diseases can also cause perfusion defects [80].

Streaky and beam-hardening effects around the rib, metallic materials, and around the high-concentration contrast agent in vessels cause false perfusion defects in an iodine map. The thoracic vein, superior vena cava, and right cardiac chambers are common locations for heterogeneous artifacts in blood flow images. The right middle lobe and left lingular segment, which abut the cardiac border, also cause heterogeneous artifacts due to cardiac motion (Fig. 8). These should not be mistaken for true lesions.

\section{Future Directions for Diagnosis of PE with DECT Using Artificial Intelligence}

Artificial intelligence (AI) has received considerable attention in the field of radiology [81]. AI can also be used to diagnose PE [82]. In the early research of Blackmon et al. [83], they developed a computer-aided detection algorithm for PE using machine learning; this algorithm
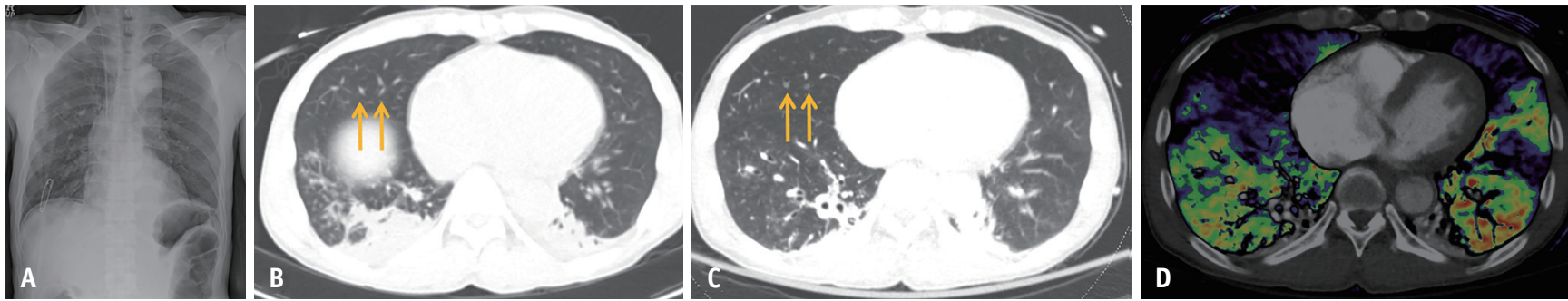

Fig. 7. A 50-year-old male suddenly complained of dyspnea after removal of a central venous catheter on day 5 after distal pancreatectomy and splenectomy.

A. Chest X-ray on day 4 after the operation showing the normal position of the central venous catheter through the right internal jugular vein. B, C. Abdominal CT on day 4 after the operation showing dependent atelectasis without embolic lesions (B). Chest CT image after dyspnea is presented (C). Multifocal air is suspected in the right middle pulmonary arteries (arrows in $\mathbf{C}$ ), which was not noted in the previous abdominal CT (arrows in B), and hypoattenuations in both anterior lungs can be seen. D. The fusion image of CT angiography and a color-coded iodine map showing large perfusion defects in the anterior portion of both lungs, which could have been caused by pulmonary air embolism. CT = computed tomography
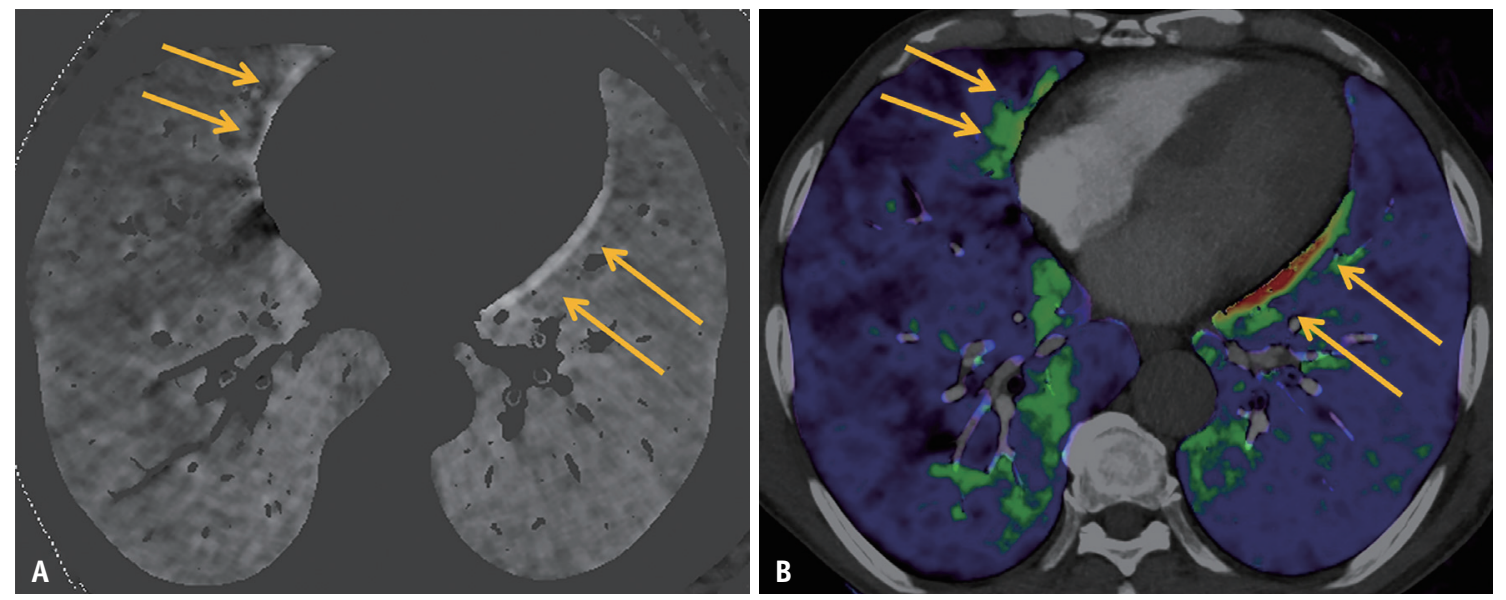

Fig. 8. A 73-year-old male diagnosed with chronic pulmonary thromboembolism.

A, B. A gray scale (A) and a fusion image iodine map (B) shows that the right middle lobe and left lingular segment (arrows) which abut the cardiac border also cause heterogeneous artifacts due to cardiac motion. 
improved the sensitivity of PE detection for inexperienced readers. In a recent study, Liu et al. [84] adapted deeplearning algorithms to detect and calculate the clot burden of acute PE using MDCT. They showed that deeplearning algorithms could detect acute PE with good performance and efficiently calculate the clot burden to reduce clinicians' workload. AI is useful for difficult tasks, such as the detection of small peripheral clots or automatic quantification of clot burden and RV/LV diameter ratio, which have been shown to be prognostic factors for acute PE. There are few studies on the application of AI in DECT imaging for PE. A recent study reported that deep learningbased automatic lung lobe segmentation and quantitative lobar or zonal perfusion analyses are useful in PE detection, although the study had a small sample size of only 88 patients [84]. Further large-scale studies applying AI to DECT images of PE are worthwhile. AI will serve to detect and characterize the emboli and predict patient prognosis by deep learning-based quantification of defect volume using DECT as a complementary diagnostic tool

\section{CONCLUSION}

DECT is useful for detecting PE, assessing the severity of $P E$, and predicting prognosis. The technical and diagnostic pitfalls of DECT have been overcome with the standardization of protocols and advancements in CT technology. According to current guidelines, DECT is not regarded as a routine diagnostic procedure in the evaluation of PE. However, DECT has been recommended as an alternative tool for $\mathrm{V} / \mathrm{Q}$ scanning in patients suspected of having CTEPH if appropriate expertise and resources are available on-site [7].

By adjusting image acquisition and post-processing methods based on clinical experience, the application of the dual-energy technique for the evaluation of PE could be expanded.

\section{Conflicts of Interest}

The authors have no potential conflicts of interest to disclose.

\section{Acknowledgments}

Our special thanks go to Ms. Sunyoung Park from Siemens Healthineers, Korea and Ms. Kyoung A Um from GE Heathcare, Korea for the support related to this work.

\section{Author Contributions}

Conceptualization: Yoo Jin Hong, Jin Hur. Data curation: Yoo Jin Hong, Jina Shim, Sang Min Lee, Dong Jin Im. Formal analysis: Yoo Jin Hong, Jin Hur. Investigation: Yoo Jin Hong, Jin Hur. Methodology: Yoo Jin Hong, Jin Hur. Software: Jina Shim, Sang Min Lee, Dong Jin Im. Supervision: Jin Hur. Validation: Jin Hur. Visualization: Yoo Jin Hong, Jina Shim. Writing - original draft: Yoo Jin Hong, Jin Hur, Sang Min Lee. Writing—review \& editing: Jin Hur.

\author{
ORCID iDs \\ Yoo Jin Hong \\ https://orcid.org/0000-0002-7276-0944 \\ Jina Shim \\ https://orcid.org/0000-0003-2041-2611 \\ Sang Min Lee \\ https://orcid.org/0000-0002-2173-2193 \\ Dong Jin Im \\ https://orcid.org/0000-0001-8139-5646 \\ Jin Hur \\ https://orcid.org/0000-0002-8651-6571
}

\section{REFERENCES}

1. Giuntini C, Di Ricco G, Marini C, Melillo E, Palla A. Pulmonary embolism: epidemiology. Chest 1995;107:3S-9S

2. Tapson VF. Acute pulmonary embolism. N Engl J Med 2008;358:1037-1052

3. Geibel A, Just H, Kasper W, Konstantinides S. Acute pulmonary embolism: a challenge for hemostasiology. Berlin: Springer Science \& Business Media, 2012

4. Ruggiero A, Screaton NJ. Imaging of acute and chronic thromboembolic disease: state of the art. Clin Radiol 2017;72:375-388

5. Heit JA. The epidemiology of venous thromboembolism in the community. Arterioscler Thromb Vasc Biol 2008;28:370-372

6. Remy-Jardin M, Pistolesi M, Goodman LR, Gefter WB, Gottschalk A, Mayo JR, et al. Management of suspected acute pulmonary embolism in the era of CT angiography: a statement from the Fleischner Society. Radiology 2007;245:315-329

7. Konstantinides SV, Meyer G, Becattini C, Bueno H, Geersing GJ, Harjola VP, et al. 2019 ESC Guidelines for the diagnosis and management of acute pulmonary embolism developed in collaboration with the European Respiratory Society (ERS). Eur Heart J 2020;41:543-603

8. Ghaye B, Szapiro D, Mastora I, Delannoy V, Duhamel A, Remy $\mathrm{J}$, et al. Peripheral pulmonary arteries: how far in the lung does multi-detector row spiral CT allow analysis? Radiology 2001;219:629-636 
9. Stein PD, Fowler SE, Goodman LR, Gottschalk A, Hales CA, Hull RD, et al. Multidetector computed tomography for acute pulmonary embolism. N Engl J Med 2006;354:2317-2327

10. Stein PD, Woodard PK, Weg JG, Wakefield TW, Tapson VF, Sostman HD, et al. Diagnostic pathways in acute pulmonary embolism: recommendations of the PIOPED II investigators. Am J Med 2006;119:1048-1055

11. Stein PD, Woodard PK, Weg JG, Wakefield TW, Tapson VF, Sostman HD, et al. Diagnostic pathways in acute pulmonary embolism: recommendations of the PIOPED II investigators. Radiology 2007;242:15-21

12. Hess S, Frary EC, Gerke 0, Madsen PH. State-of-the-art imaging in pulmonary embolism: ventilation/perfusion singlephoton emission computed tomography versus computed tomography angiography-controversies, results, and recommendations from a systematic review. Semin Thromb Hemost 2016;42:833-845

13. Kang DK, Thilo C, Schoepf UJ, Barraza JM Jr, Nance JW Jr, Bastarrika $\mathrm{G}$, et al. CT signs of right ventricular dysfunction: prognostic role in acute pulmonary embolism. JACC CardiovasC Imaging 2011;4:841-849

14. Vedovati MC, Becattini C, Agnelli G, Kamphuisen PW, Masotti L, Pruszczyk $\mathrm{P}$, et al. Multidetector CT scan for acute pulmonary embolism: embolic burden and clinical outcome. Chest 2012;142:1417-1424

15. Meinel FG, Nance JW Jr, Schoepf UJ, Hoffmann VS, Thierfelder KM, Costello $P$, et al. Predictive value of computed tomography in acute pulmonary embolism: systematic review and meta-analysis. Am J Med 2015;128:747-759.e2

16. Johnson TR, Krauss B, Sedlmair M, Grasruck M, Bruder H, Morhard D, et al. Material differentiation by dual energy CT: initial experience. Eur Radiol 2007;17:1510-1517

17. Goo HW, Goo JM. Dual-energy CT: new horizon in medical imaging. Korean J Radiol 2017;18:555-569

18. Danad I, Fayad ZA, Willemink MJ, Min JK. New applications of cardiac computed tomography: dual-energy, spectral, and molecular CT imaging. JACC Cardiovasc Imaging 2015;8:710723

19. Rassouli N, Etesami M, Dhanantwari A, Rajiah P. Detectorbased spectral CT with a novel dual-layer technology: principles and applications. Insights Imaging 2017;8:589-598

20. Thieme SF, Johnson TR, Lee C, McWilliams J, Becker CR, Reiser $M F$, et al. Dual-energy CT for the assessment of contrast material distribution in the pulmonary parenchyma. AJR Am J Roentgenol 2009;193:144-149

21. Lu GM, Wu SY, Yeh BM, Zhang LJ. Dual-energy computed tomography in pulmonary embolism. Br J Radiol 2010;83:707718

22. Pontana F, Faivre JB, Remy-Jardin M, Flohr T, Schmidt $B$, Tacelli $N$, et al. Lung perfusion with dual-energy multidetector-row CT (MDCT): feasibility for the evaluation of acute pulmonary embolism in 117 consecutive patients. Acad Radiol 2008;15:1494-1504

23. Thieme SF, Hoegl S, Nikolaou K, Fisahn J, Irlbeck M, Maxien D, et al. Pulmonary ventilation and perfusion imaging with dualenergy CT. Eur Radiol 2010;20:2882-2889

24. Geyer LL, Scherr M, Körner M, Wirth S, Deak P, Reiser MF, et al. Imaging of acute pulmonary embolism using a dual energy CT system with rapid kVp switching: initial results. Eur J Radiol 2012;81:3711-3718

25. Fink C, Johnson TR, Michaely HJ, Morhard D, Becker C, Reiser $M$, et al. Dual-energy CT angiography of the lung in patients with suspected pulmonary embolism: initial results. Rofo 2008;180:879-883

26. Weidman EK, Plodkowski AJ, Halpenny DF, Hayes SA, PerezJohnston R, Zheng J, et al. Dual-energy CT angiography for detection of pulmonary emboli: incremental benefit of iodine maps. Radiology 2018;289:546-553

27. Zhang LJ, Zhao YE, Wu SY, Yeh BM, Zhou CS, Hu XB, et al. Pulmonary embolism detection with dual-energy CT: experimental study of dual-source CT in rabbits. Radiology 2009;252:61-70

28. Thieme SF, Ashoori N, Bamberg F, Sommer WH, Johnson TR, Leuchte $\mathrm{H}$, et al. Severity assessment of pulmonary embolism using dual energy CT - correlation of a pulmonary perfusion defect score with clinical and morphological parameters of blood oxygenation and right ventricular failure. Eur Radiol 2012;22:269-278

29. Thieme SF, Becker CR, Hacker M, Nikolaou K, Reiser $M F$, Johnson TR. Dual energy CT for the assessment of lung perfusion--correlation to scintigraphy. Eur J Radiol 2008;68:369-374

30. Wu HW, Cheng JJ, Li JY, Yin Y, Hua J, Xu JR. Pulmonary embolism detection and characterization through quantitative iodine-based material decomposition images with spectral computed tomography imaging. Invest Radiol 2012;47:85-91

31. Chai X, Zhang LJ, Yeh BM, Zhao YE, Hu XB, Lu GM. Acute and subacute dual energy CT findings of pulmonary embolism in rabbits: correlation with histopathology. $\mathrm{Br} J$ Radiol 2012;85:613-622

32. Wu AS, Pezzullo JA, Cronan JJ, Hou DD, Mayo-Smith WW. CT pulmonary angiography: quantification of pulmonary embolus as a predictor of patient outcome--initial experience. Radiology 2004;230:831-835

33. Dournes $G$, Verdier D, Montaudon M, Bullier E, Rivière A, Dromer $C$, et al. Dual-energy CT perfusion and angiography in chronic thromboembolic pulmonary hypertension: diagnostic accuracy and concordance with radionuclide scintigraphy. Eur Radiol 2014;24:42-51

34. Meyer M, Haubenreisser H, Sudarski S, Doesch C, Ong MM, Borggrefe $M$, et al. Where do we stand? Functional imaging in acute and chronic pulmonary embolism with state-of-the-art CT. Eur J Radiol 2015;84:2432-2437

35. Okada M, Kunihiro Y, Nakashima Y, Nomura T, Kudomi S, Yonezawa $\mathrm{T}$, et al. Added value of lung perfused blood volume images using dual-energy CT for assessment of acute pulmonary embolism. Eur J Radiol 2015;84:172-177

36. Kröger JR, Hickethier T, Pahn G, Gerhardt F, Maintz D, Bunck 
AC. Influence of spectral detector CT based monoenergetic images on the computer-aided detection of pulmonary artery embolism. Eur J Radiol 2017;95:242-248

37. Singh R, Nie RZ, Homayounieh F, Schmidt B, Flohr T, Kalra MK. Quantitative lobar pulmonary perfusion assessment on dualenergy $\mathrm{CT}$ pulmonary angiography: applications in pulmonary embolism. Eur Radiol 2020;30:2535-2542

38. Kong X, Sheng HX, Lu GM, Meinel FG, Dyer KT, Schoepf UJ, et al. Xenon-enhanced dual-energy $C T$ lung ventilation imaging: techniques and clinical applications. AJR Am J Roentgenol 2014;202:309-317

39. Delesalle MA, Pontana F, Duhamel A, Faivre JB, Flohr T, Tacelli $\mathrm{N}$, et al. Spectral optimization of chest $\mathrm{CT}$ angiography with reduced iodine load: experience in 80 patients evaluated with dual-source, dual-energy CT. Radiology 2013;267:256-266

40. Chae EJ, Seo JB, Goo HW, Kim N, Song KS, Lee SD, et al. Xenon ventilation $\mathrm{CT}$ with a dual-energy technique of dualsource CT: initial experience. Radiology 2008;248:615-624

41. Zhang LJ, Zhou CS, Schoepf UJ, Sheng HX, Wu SY, Krazinski AW, et al. Dual-energy CT lung ventilation/perfusion imaging for diagnosing pulmonary embolism. Eur Radiol 2013;23:26662675

42. Hong SR, Chang S, Im DJ, Suh YJ, Hong YJ, Hur J, et al. Feasibility of single scan for simultaneous evaluation of regional krypton and iodine concentrations with dual-energy CT: an experimental study. Radiology 2016;281:597-605

43. Chang S, Hur J, Im DJ, Suh YJ, Hong YJ, Lee HJ, et al. Dualenergy CT-based iodine quantification for differentiating pulmonary artery sarcoma from pulmonary thromboembolism: a pilot study. Eur Radiol 2016;26:3162-3170

44. Ito K, Kubota K, Morooka M, Shida Y, Hasuo K, Endo H, et al. Diagnostic usefulness of 18F-FDG PET/CT in the differentiation of pulmonary artery sarcoma and pulmonary embolism. Ann Nucl Med 2009;23:671-676

45. Lee EJ, Moon SH, Choi JY, Lee KS, Choi YS, Choe YS, et al. Usefulness of fluorodeoxyglucose positron emission tomography in malignancy of pulmonary artery mimicking pulmonary embolism. ANZ J Surg 2013;83:342-347

46. Xi XY, Gao W, Gong JN, Guo XJ, Wu JY, Yang YH, et al. Value of 18F-FDG PET/CT in differentiating malignancy of pulmonary artery from pulmonary thromboembolism: a cohort study and literature review. Int J Cardiovasc Imaging 2019;35:1395-1403

47. Chong S, Kim TS, Kim BT, Cho EY, Kim J. Pulmonary artery sarcoma mimicking pulmonary thromboembolism: integrated FDG PET/CT. AJR Am J Roentgenol 2007;188:1691-1693

48. Kamel EM, McKee TA, Calcagni ML, Schmidt S, Markl S, Castaldo $S$, et al. Occult lung infarction may induce false interpretation of $18 \mathrm{~F}-$ FDG PET in primary staging of pulmonary malignancies. Eur J Nucl Med Mol Imaging 2005;32:641-646

49. Wittram C, Scott JA. 18F-FDG PET of pulmonary embolism. AJR Am J Roentgenol 2007;189:171-176

50. Collomb D, Paramelle PJ, Calaque 0, Bosson JL, Vanzetto G, Barnoud D, et al. Severity assessment of acute pulmonary embolism: evaluation using helical CT. Eur Radiol
2003;13:1508-1514

51. van der Meer RW, Pattynama PM, van Strijen MJ, van den Berg-Huijsmans AA, Hartmann IJ, Putter $\mathrm{H}$, et al. Right ventricular dysfunction and pulmonary obstruction index at helical CT: prediction of clinical outcome during 3-month follow-up in patients with acute pulmonary embolism. Radiology 2005;235:798-803

52. Apfaltrer P, Bachmann V, Meyer M, Henzler T, Barraza JM, Gruettner J, et al. Prognostic value of perfusion defect volume at dual energy CTA in patients with pulmonary embolism: correlation with CTA obstruction scores, CT parameters of right ventricular dysfunction and adverse clinical outcome. Eur J Radiol 2012;81:3592-3597

53. Bauer RW, Frellesen C, Renker M, Schell B, Lehnert T, Ackermann $\mathrm{H}$, et al. Dual energy CT pulmonary blood volume assessment in acute pulmonary embolism - correlation with D-dimer level, right heart strain and clinical outcome. Eur Radiol 2011;21:1914-1921

54. Chae EJ, Seo JB, Jang YM, Krauss B, Lee CW, Lee HJ, et al. Dual-energy $C T$ for assessment of the severity of acute pulmonary embolism: pulmonary perfusion defect score compared with $\mathrm{CT}$ angiographic obstruction score and right ventricular/left ventricular diameter ratio. AJR Am J Roentgenol 2010;194:604-610

55. Kong WF, Wang YT, Yin LL, Pu H, Tao KY. Clinical risk stratification of acute pulmonary embolism: comparing the usefulness of CTA obstruction score and pulmonary perfusion defect score with dual-energy CT. Int J Cardiovasc Imaging 2017;33:2039-2047

56. Meinel FG, Graef A, Bamberg F, Thieme SF, Schwarz F, Sommer WH, et al. Effectiveness of automated quantification of pulmonary perfused blood volume using dual-energy CTPA for the severity assessment of acute pulmonary embolism. Invest Radiol 2013;48:563-569

57. Takx RAP, Henzler T, Schoepf UJ, Germann T, Schoenberg S0, Shirinova A, et al. Predictive value of perfusion defects on dual energy CTA in the absence of thromboembolic clots. $J$ Cardiovasc Comput Tomogr 2017;11:183-187

58. Im DJ, Hur J, Han KH, Lee HJ, Kim YJ, Kwon W, et al. Acute pulmonary embolism: retrospective cohort study of the predictive value of perfusion defect volume measured with dual-energy CT. AJR Am J Roentgenol 2017;209:1015-1022

59. Im DJ, Hur J, Han K, Suh YJ, Hong YJ, Lee HJ, et al. Prognostic value of dual-energy CT-based iodine quantification versus conventional $\mathrm{CT}$ in acute pulmonary embolism: a propensity-match analysis. Korean J Radiol 2020;21:10951103

60. Remy-Jardin M, Duhamel A, Deken V, Bouaziz N, Dumont P, Remy J. Systemic collateral supply in patients with chronic thromboembolic and primary pulmonary hypertension: assessment with multi-detector row helical CT angiography. Radiology 2005;235:274-281

61. Hong YJ, Kim JY, Choe K0, Hur J, Lee HJ, Choi BW, et al. Different perfusion pattern between acute and chronic 
pulmonary thromboembolism: evaluation with two-phase dual-energy perfusion CT. AJR Am J Roentgenol 2013;200:812817

62. Kim SS, Hur J, Kim YJ, Lee HJ, Hong YJ, Choi BW. Dualenergy $\mathrm{CT}$ for differentiating acute and chronic pulmonary thromboembolism: an initial experience. Int J Cardiovasc Imaging 2014;30 Suppl 2:113-120

63. Yang S, Yang Y, Zhai Z, Kuang T, Gong J, Zhang S, et al. Incidence and risk factors of chronic thromboembolic pulmonary hypertension in patients after acute pulmonary embolism. J Thorac Dis 2015;7:1927-1938

64. Vavera Z, Vojacek J, Pudil R, Maly J, Elias P. Chronic thromboembolic pulmonary hypertension after the first episode of pulmonary embolism? How often? Biomed Pap Med Fac Univ Palacky Olomouc Czech Repub 2016;160:125-129

65. Poli $D$, Miniati M. The incidence of recurrent venous thromboembolism and chronic thromboembolic pulmonary hypertension following a first episode of pulmonary embolism. Curr Opin Pulm Med 2011;17:392-397

66. Dentali F, Donadini M, Gianni M, Bertolini A, Squizzato A, Venco $A$, et al. Incidence of chronic pulmonary hypertension in patients with previous pulmonary embolism. Thromb Res 2009;124:256-258

67. Becattini C, Agnelli G, Pesavento R, Silingardi M, Poggio $R$, Taliani $M R$, et al. Incidence of chronic thromboembolic pulmonary hypertension after a first episode of pulmonary embolism. Chest 2006;130:172-175

68. Pengo V, Lensing AW, Prins MH, Marchiori A, Davidson BL, Tiozzo F, et al. Incidence of chronic thromboembolic pulmonary hypertension after pulmonary embolism. $N$ Engl J Med 2004;350:2257-2264

69. Piazza G, Goldhaber SZ. Chronic thromboembolic pulmonary hypertension. N Engl J Med 2011;364:351-360

70. Giordano J, Khung S, Duhamel A, Hossein-Foucher C, Bellèvre $D$, Lamblin $\mathrm{N}$, et al. Lung perfusion characteristics in pulmonary arterial hypertension (PAH) and peripheral forms of chronic thromboembolic pulmonary hypertension ( $\mathrm{pCTEPH)}$ : dual-energy CT experience in 31 patients. Eur Radiol 2017;27:1631-1639

71. Takagi H, Ota H, Sugimura K, Otani K, Tominaga J, Aoki T, et al. Dual-energy CT to estimate clinical severity of chronic thromboembolic pulmonary hypertension: comparison with invasive right heart catheterization. Eur J Radiol 2016;85:1574-1580

72. Henzler T, Fink C, Schoenberg S0, Schoepf UJ. Dualenergy CT: radiation dose aspects. AJR Am J Roentgenol 2012;199:S16-S25

73. Schenzle JC, Sommer WH, Neumaier K, Michalski G, Lechel U, Nikolaou K, et al. Dual energy CT of the chest: how about the dose? Invest Radiol 2010;45:347-353

74. Matsubara K, Takata T, Kobayashi M, Kobayashi S, Koshida K, Gabata T. Tube current modulation between single-and dualenergy CT with a second-generation dual-source scanner: radiation dose and image quality. AJR Am J Roentgenol
2016;207:354-361

75. Krauss B, Grant KL, Schmidt BT, Flohr TG. The importance of spectral separation: an assessment of dual-energy spectral separation for quantitative ability and dose efficiency. Invest Radiol 2015;50:114-118

76. Lenga L, Leithner D, Peterke JL, Albrecht MH, Gudauskas T, D'Angelo $\mathrm{T}$, et al. Comparison of radiation dose and image quality of contrast-enhanced dual-source CT of the chest: single-versus dual-energy and second-versus third-generation technology. AJR Am J Roentgenol 2019;212:741-747

77. Lenga L, Trapp F, Albrecht MH, Wichmann JL, Johnson AA, Yel I, et al. Single- and dual-energy CT pulmonary angiography using second- and third-generation dual-source CT systems: comparison of radiation dose and image quality. Eur Radiol 2019;29:4603-4612

78. Lu GM, Zhao Y, Zhang LJ, Schoepf UJ. Dual-energy CT of the lung. AJR Am J Roentgenol 2012;199:S40-S53

79. Ikeda Y, Yoshimura N, Hori Y, Horii Y, Ishikawa H, Yamazaki $M$, et al. Analysis of decrease in lung perfusion blood volume with occlusive and non-occlusive pulmonary embolisms. Eur J Radiol 2014;83:2260-2267

80. Kim BH, Seo JB, Chae EJ, Lee HJ, Hwang HJ, Lim C. Analysis of perfusion defects by causes other than acute pulmonary thromboembolism on contrast-enhanced dual-energy CT in consecutive 537 patients. Eur J Radiol 2012;81:e647-e652

81. Hosny A, Parmar C, Quackenbush J, Schwartz LH, Aerts HJWL. Artificial intelligence in radiology. Nat Rev Cancer 2018;18:500-510

82. Remy-Jardin M, Faivre JB, Kaergel R, Hutt A, Felloni P, Khung $S$, et al. Machine learning and deep neural network applications in the thorax: pulmonary embolism, chronic thromboembolic pulmonary hypertension, aorta, and chronic obstructive pulmonary disease. J Thorac Imaging 2020;35 Suppl 1:S40-S48

83. Blackmon KN, Florin C, Bogoni L, McCain JW, Koonce JD, Lee $\mathrm{H}$, et al. Computer-aided detection of pulmonary embolism at CT pulmonary angiography: can it improve performance of inexperienced readers? Eur Radiol 2011;21:1214-1223

84. Liu W, Liu M, Guo X, Zhang P, Zhang L, Zhang R, et al. Evaluation of acute pulmonary embolism and clot burden on CTPA with deep learning. Eur Radiol 2020;30:3567-3575

85. Zhang LJ, Yang GF, Zhao YE, Zhou CS, Lu GM. Detection of pulmonary embolism using dual-energy computed tomography and correlation with cardiovascular measurements: a preliminary study. Acta Radiol 2009;50:892-901

86. Lee CW, Seo JB, Song JW, Kim MY, Lee HY, Park YS, et al. Evaluation of computer-aided detection and dual energy software in detection of peripheral pulmonary embolism on dual-energy pulmonary CT angiography. Eur Radiol 2011;21:54-62

87. Zhang J, Cai J, Liu S, Zhang X. Value of dual-energy lung perfusion imaging using a dual-source $\mathrm{CT}$ system for the pulmonary embolism. Open Life Sci 2018;13:107-111

88. Nakazawa T, Watanabe Y, Hori Y, Kiso K, Higashi M, Itoh T, 
et al. Lung perfused blood volume images with dual-energy computed tomography for chronic thromboembolic pulmonary hypertension: correlation to scintigraphy with single-photon emission computed tomography. J Comput Assist Tomogr 2011;35:590-595
89. Masy M, Giordano J, Petyt G, Hossein-Foucher C, Duhamel $A$, Kyheng $M$, et al. Dual-energy CT (DECT) lung perfusion in pulmonary hypertension: concordance rate with $\mathrm{V} / \mathrm{Q}$ scintigraphy in diagnosing chronic thromboembolic pulmonary hypertension (CTEPH). Eur Radiol 2018;28:5100-5110 